\title{
Development of a Robotic Hand Orthosis for Stroke Patient Rehabilitation
}

\author{
https://doi.org/10.3991/ijoe.v16i13.13407
}

\author{
Emmanuel Osayande, Kayode P. Ayodele ${ }^{(凶)}$, Morenikeji A. Komolafe \\ Obafemi Awolowo University, Ile-Ife, Nigeria \\ kayodele@oauife.edu.ng
}

\begin{abstract}
This study developed a robotic orthosis capable of detecting the intention of a wearer to move their fingers, thereafter augmenting their muscle force. This was with the aim of producing a device that can be used in post-stroke hand rehabilitation. The design of the orthosis was based on an existing design, which was modified using BLENDER release 2.78 and printed with ABS plastic. An actuator was mounted at the rear end of the orthosis, to provide actuation to perform full range flexion and extension motion for digits. Force sensors were embedded at the fingertips of the orthosis to detect minute finger movements. For severe cases where stroke survivors are incapable of little finger movements, the study employed a brain-computer interface to detect the intent to move. The robotic orthosis achieved an accuracy of $64.1 \%$ and $62 \%$ in detecting unclench and clench activities respectively and actuating the orthosis digits in response. The results revealed that the design presented here can help provide effective hand rehabilitation. The study concluded that the design incorporated with BCI systems is capable of performing hand rehabilitation in a clinical setting as it obtains some level of accuracy in detecting patient intent to move and actuating in response. This design is low cost, and hence will lessen the economic burden for stroke survivors in a poor-resource country.
\end{abstract}

Keywords - Stroke, robotic rehabilitation, brain-computer interface, orthosis

\section{Introduction}

A stroke is caused by the interruption of the blood supply to parts of the brain, usually because a blood vessel bursts or is blocked by a clot [1]. Stroke is one of the leading causes of neurological impairment or motor disabilities worldwide. Permanent neurological impairment occurs in about 600 million people, with most of them elderly [2]. Even though an increased effort is made on the recovery process of stroke patients, the deficiency of available human resources and economic constraints means that often patients do not attain full recovery when discharged from hospital after rehabilitation. This has a great negative impact on the quality of life of the stroke survivors. However, there are indications that specially-designed exercises may equally expand neuroplasticity and therapeutic outcomes both during the severe phase immediately after the stroke and in the long run [3]. 
A lot of attention is therefore given to post-stroke rehabilitation, towards partial or total recovery of motor functions. These treatment methods include intense task-directed training, treadmill training, aerobic exercises [2], passive facilitation, promotion of alternative movements, [4], constraint-induced movement therapy, [2]. Other strategies based on neurophysiological learning mechanisms to promote neuroplasticity include robot-assisted therapy [5] reinforced feedback exercises in a virtual environment, [2] and recently brain-computer interface (BCI) [6].

Although numerous studies have presented evidence that recovery of motor functions is probable, there remain several challenges that are associated with the current practice where physical therapy is administered by a physiotherapist. These challenges include slow recovery time as a result of less frequent physical practice and intensity, a large number of resources and time is required, the patient will have to frequently transit to clinics bringing discomforts for patients, cost of therapy is usually high and therapist is completely dedicated to a single patient.

All these challenges are present because effective rehabilitation depends on some factors some of which are: high-intensity practice [7-8], mental practice with motor imagery, moving platform training [9] and task-specific training. The most effective rehabilitation approaches have been found to be those where specific functional tasks are practiced repeatedly [4]. Robotic rehabilitation seems to provide adequate solutions to these problems and challenges. Since such intensive training is no longer cost-effective and requires a significant amount of relentless effort, robotic rehabilitation devices (RRD) have been introduced to aid the therapist in providing consistent, repeatable training with less effort [8]. With many of these systems showing therapy outcomes comparable to equivalent intensive training without robotic aid [5] or an equal number of unassisted movements [3], this suggests that one of the primary benefits of robotic training is its ability to provide intensive therapy at a lower cost with less effort.

In the present study, a robotic orthosis was developed with the aim of eventually investigating the possibility of using it to reduce post-stroke recovery of hand motor function. The orthosis detects the intention of a user to move their fingers, and augments their muscle force. To cater to users who are incapable of summoning enough force to be detected by the orthosis sensors, an electroencephalography (EEG)-based system is used to infer movement intent from scalp-acquired brain waves.

\section{Methods}

\subsection{Methodology overview}

A block diagram of the orthosis is presented in Fig. 1. The processes involved both hardware development, software analysis. The hardware development consists of 3D design and construction of a hand orthosis shell, while the software involved various analyses form the pre-processing of the EEG data to the point of classification and evaluation. 


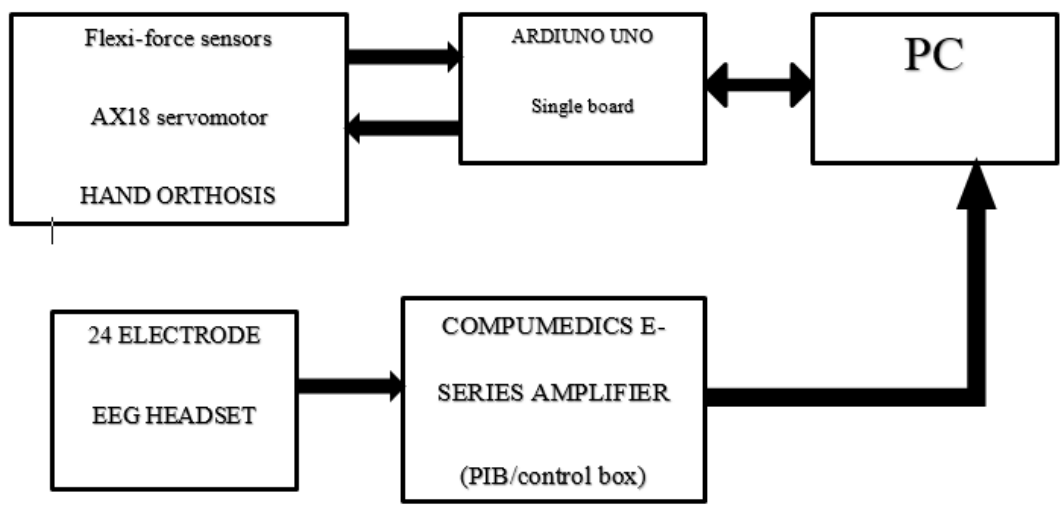

Fig. 1. Block diagram of the instrumentation and control system

\subsection{The hand orthosis design}

The mechanical design of the orthosis was based on an existing 3D design [10] which consists of 59 individual parts. This design was modified using release 2.79 of the Blender 3D modelling package. The modification was based on three things; inclusion of support structures for the motors to achieve full range of motion of the digits, the user's comfort and wear-ability. The design was started with a plaster cast that was made of a human subject hand which was then filled with liquid ceramic mass with some metal support structure inside, making the model easier to hold and observe. The hand's model was now smoothened and polished, which was later scanned by a 3D scanner. Closely fitted to the wrist is the husk of the orthosis which was necessary to for the orthosis to be well-placed on the hand, while the fingers were supported by smaller parts emulating their joint-structure; the MCP (Metacarpaphalangeal) and DIP (Distal Interphalangeal) joints for the thumb and MCP, PIP (Proximal Interphalangeal) and DIP joints in every other finger.

To completely integrate all the 59 individual parts into a final working piece, some lever mechanism was put in place and situated at the top of the husk. Applying a forward force to the main lever mechanism allows the orthosis to perform the grasping motion and pulling away the lever returns the hand to its resting state. After all necessary modification, the parts were all exported as STL files. Cross-checking for any discrepancies was done before finally importing to CURA software to prepare for 3D printing.

Fabrication was done on an Ultimaker 2 3D printer with extruder head of $0.4 \mathrm{~mm}$. Printing was done with acrylonitrile butadiene styrene (ABS) filaments of different colors. Small 3D printed pins were used to join the smaller leverages and finger sleeves to each other while the main lever was coupled to the husk with the 3D printed latches. A strap was introduced to fasten the orthosis around the subject's hand. 


\subsection{The hand orthosis instrumentation}

This section describes the various instrumentation used in the hand orthosis development. These are software and hardware instruments which includes the force sensitive resistor, Arduino Uno and the actuator (Dynamixel motors AX18).

A force-sensitive resistor (FSR) was chosen for this research work. The hardware setup involved creating a voltage divider with the FSR and another resistor $(220 \Omega)$, hence creating a variable voltage output, which was fed into a microcontroller ADC input which is mounted on the Arduino Uno. The choice of a static resistor was based on sensitivity and stability, and also to maintain the threshold of the maximum resistance and minimum resistance of the FSR. A simple code is written in C\# to calculate the applied force. The data collected from the FSR was subsequently used to control the orthosis motion for flexion and extension.

An AX18 servomotor was placed on the part of the orthosis that sits on the fore-arm (the husk), this is done in a bid to maintain the light weight of the orthosis. The actuator was laid horizontally with its gear head facing the lever mechanism of the orthosis and connected to a lead screw mechanism to convert the rotational motion into translational motion. A lead screw mechanism converts rotational motion to linear motion and a torque (rotational force) to linear force [11]. It basically consists of a screw and nut; the screw passes through the nut with threads on the inside of the nut that mesh with the threads on the screw. When the shaft of the stationary screw is rotated relative to the thread of the nut, the nut moves along its axis relative to the medium surrounding it. Therefore, as the actuator gear head rotates it translate the nut in a forward or backward motion which then pushes the lever attached to the finger compartments of the robotic orthosis when it moves forward and when it moves backward it pulls on the lever. The FSR was placed at the fingertips of the robotic orthosis to sense minute finger movement.

\subsection{BCI instrumentation}

This section briefly describes various instrumentation used for the BCI experimental procedures. These are hardware and software instruments which include the Compumedics E-series patient interface box amplifier for anti-aliasing filtering and amplification, OpenSesame for stimulus presentation and adjustable headset and electrodes, which served as an interface between the scalp and the amplifier.

The E-series patient interface box (PIB) is a medical grade EEG acquisition system, which provides up 32 touch-proof connectors for patient electrode inputs and a cable connection to the control module. The PIB amplifies, filters and digitizes the patient EEG signals. The PIB also provides impedance testing and calibration functions. The headsets are made of elastic rubber material, which ensured that the headset could adapt to any head size. This design also made it easy for electrodes to be easily detached for cleaning after each session. The electrodes were wet $\mathrm{Ag} / \mathrm{AgCl}$ placed in accordance to the 10-20 system of electrodes arrangement. 
To present cues to the experiment subjects to perform imagined movement for BCI control at a particular time, a stimulus presentation designed using OpenSesame (version 3.1.9), which is a stimulus presentation software used in psychological experiments. To match the cue to clench and unclench the hand with the recorded EEG data, a video recording of the stimulus presentation was done. The start of the recording was synchronized with the start of the experiment, thereafter a multimedia framework software (known as FFMPEG) was used to extract each frame in the video with a timestamp. This helps to mark the EEG data at the onset of the cues with some fair accuracy.

\subsection{Signal acquisition}

This is the process of extracting signals from BCI subjects using electrode headset and amplifier. The paradigm followed for this experiment made use of the real hand movement of the subjects for decoding of flexion (opening) or extension (closing) movement of the hand. In this experiment, flexion and extension hand movements were considered and specified for the grasping tasks to eliminate other variables due to movements such as the elbow joint movement and shoulder movements. Alongside, the subjects were restrained to an adjustable upright sitting position against a straight back and comfortable armless chair with no head movement allowed or eye blinking allowed to help eliminate artifacts caused by these movements.

Three healthy subjects with ages between 18 and 30 years and three stroke subjects between 21 and 85 years took part in the experiment, and they all gave their written informed consent to the study. The healthy subjects were all selected at random from the student population of Obafemi Awolowo University, while the stroke subjects were selected from the Obafemi Awolowo University Teaching Hospital Neurological clinic and Physiotherapy clinic as well. None of them had previous experiences with BCI. The ethics committee of Obafemi Awolowo University Ile-Ife approved the experiment.

The experiment was conducted using the E-series Compumedics data acquisition hardware with 16-bit resolution, 24 channels and referential montage wet electrodes $(\mathrm{Ag} / \mathrm{AgCl})$ with impedance maintained at less than $10 \mathrm{k} \Omega$. Sampling rate at $256 \mathrm{~Hz}$, with low pass filters at $30 \mathrm{~Hz}$, high pass filters at $1 \mathrm{~Hz}$ and Notch filter set at $50 \mathrm{~Hz}$. A total of 100 single trials of EEG that randomly comprises motor imagery of the strokeaffected upper limb during the flexion and extension movement was collected and also EEG for background rest condition was collected. In the experiment, OpenSesame software was used to cue the subjects to perform motor imagery. The 100 trials were broken into 2 runs [12 -13] that consist of 50 trials each with at least five minutes break after each run, this helped reduce fatigue and improve subject concentration. Each trial lasted for approximately $12 \mathrm{~s}$, and the entire 100 trials lasted for about 30mins with breaks inclusive. For each run, OpenSesame was used to prepare the subject. First, the subject was prepared with a visual cue for $2 \mathrm{~s}$ and another visual cue then instructs the subject to perform motor imagery or motor execution for $4 \mathrm{~s}$, followed by $6 \mathrm{~s}$ of rest. The subjects were advised to minimize any body movement and eye blinking throughout the process. 


\subsection{Data processing}

The approach used in this study was similar to that used in [14] and [15] to learn representations from the EEG data acquired. The approach is inspired by the fact that computational neural networks have regularly achieved excellent classification performance on images. If some way can be found to generate images from feature vectors, then a standard architecture can be used for simple binary classification. Consequently, for this study, the EEG data were converted into images, and subsequently classified using a VGG-style architecture.

First, we transform EEG activities into series of images, as opposed to standard EEG analysis techniques. Next, a deep recurrent convolutional network is train inspired by state-of-the-art video classification techniques to learn robust representations from the series of images. Overall, the approach combines the ConvNet and RNN architecture (which is referred to RCNN) and it is aimed at preserving the spatial, spectral, and temporal structure of EEG data, and to extract features which are scaling, translation and distortion invariant. The data used in these stages had been synchronized and structured and converted into a readable format (EDF+ file) for easier access by a wider range of libraries.

\section{Results and Discussion}

The use of ABS plastic and light-weight materials ensured that the orthosis was quite light, and comfortable to wear. Table 1 shows the weight distribution of the orthosis parts.

Table 1. Robotic orthosis component mass

\begin{tabular}{|l|c|}
\hline \multicolumn{1}{|c|}{ Component } & Mass (g) \\
\hline 3D printed orthosis & 186 \\
\hline Actuator & 55.9 \\
\hline Lead screw mechanism & 14 \\
\hline Total & 255.9 \\
\hline
\end{tabular}

For the EEG data, some of the single trials were removed due to some artifacts present in the EEG data or inaccurate synchronization of the onset and offset of the cue with data recording. As a result, some subject sessions were less than 100 trials. Implementing the Barshivan approach [14] on the EEG signals acquired from the Subjects in the first session, the highest classification accuracy of $64.1 \%$ and $56.4 \%$ was achieved for the healthy subjects and stroke subjects respectively as seen in Table 2 .

It was observed that there was an improvement when the classification accuracy of stroke subjects brain wave activity for both sessions are compared. The first session achieved a classification accuracy of $55.1 \%$ compared with the accuracy of $56.4 \%$ for stroke subjects in the second session as seen in Table 2. This result shows that with more sessions and practice subjects will be able to use $\mathrm{BCI}$ better and improve classification accuracy. 
Table 2. Classification results

\begin{tabular}{|l|c|c|c|c|}
\hline \multirow{2}{*}{ Subject } & \multicolumn{3}{|c|}{ Accuracy (\%) } \\
\cline { 2 - 5 } & \multicolumn{2}{|c|}{ Session 1 } & \multicolumn{2}{c|}{ Session 2 } \\
\cline { 2 - 5 } & Clench & Unclench & Clench & Unclench \\
\hline Healthy Subject AA & $62 \%$ & $59 \%$ & $59.6 \%$ & $59.4 \%$ \\
\hline Healthy Subject AB & $53.2 \%$ & $64.1 \%$ & $60.1 \%$ & $62.3 \%$ \\
\hline Healthy Subject AC & $51.3 \%$ & $58.1 \%$ & $52.4 \%$ & $58.7 \%$ \\
\hline Stroke Subject S1 & $52.2 \%$ & $52.7 \%$ & $55.1 \%$ & $56.4 \%$ \\
\hline Stroke Subject S2 & $53.9 \%$ & $49.7 \%$ & $54.3 \%$ & $53.4 \%$ \\
\hline Stroke Subject S3 & $55.4 \%$ & $51.0 \%$ & $55.3 \%$ & $53.1 \%$ \\
\hline
\end{tabular}

\section{Conclusion}

The robotic orthosis developed yields to the basic robot therapy (RT) principle for effective rehabilitation, which is to provide intensive repetitive training with little to no supervision [16]. The orthosis also does not fatigue and can, therefore, support an intensive program, of greater repetitions and longer treatment sessions which will, therefore, reduce the time course of recovery. This design is low cost, and hence will lessen the economic burden for stroke survivors in a poor resource country.

\section{Acknowledgement}

The authors would like to thank the Opey Akinlolu foundation, Lagos for supporting this work. The workstation used for the machine learning model used a Titan Xp GPU donated by Nvidia Corporation. This Research was funded by the TET Fund National Research Fund.

\section{References}

[1] WHO (2015): "WHO Stroke, Cerebrovascular accident" [online] Available: https://www.who.int/topics/cerebrovascular_accident/en/, (Last accessed: Mar. 1, 2019)

[2] Silvoni, S., Ramos-Murguialday, A., Cavinato, M., Volpato, C., Cisotto, G., Turolla, A., Piccione, F., and Birbaumer, N. (2011): "Brain-computer interface in stroke: A review of progress.” Clinical EEG and Neuroscience, Vol. 42, No. 4, , pp. 245-252. https://doi.org/ $\underline{10.1177 / 155005941104200410}$

[3] Blank, A. A., French, J. A., and Malley, M. K. O. (2015): "Rehabilitation: Promoting Patient Engagement in Therapy.” Journal of Rehabilitation Medicine, Vol. 2, No. 3, , pp. 184-195.

[4] Masiero, S., Poli, P., Rosati, G., Zanotto, D., Iosa, M., Paolucci, S., and Morone, G. (2014): "The value of robotic systems in stroke rehabilitation." Expert Review of Medical Devices, Vol. 11, No. 2, pp. 187-198. https://doi.org/10.1586/17434440.2014.882766

[5] Norouzi-Gheidari, N., Archambault, P. S., and Fung, J. (2012): "Effects of robot-assisted therapy on stroke rehabilitation in upper limbs: systematic review and meta-analysis of the literature" Journal of rehabilitation research and development, Vol. 49, No. 4, pp. 479-96. https://doi.org/10.1682/jrrd.2010.10.0210 
[6] Ang, K. K., and Guan, C. (2015): "Brain-computer interface for neurorehabilitation of upper limb after stroke" Proceedings of the IEEE, Vol. 103, No. 6 pp. 944-953. https://doi.org/10. 1109/jproc.2015.2415800

[7] Legg, L., Pollock, A., Langhorne, P., and Sellars, P. (2000): “A multidisciplinary research agenda for stroke rehabilitation.” Br Journal of Therapy Rehabilitation, Vol. 7, , pp. 31924. https://doi.org/10.12968/bjtr.2000.7.7.13869

[8] Takahashi, C. D., Der-Yeghiaian, L., Le, V., Motiwala, R. R., and Cramer, S. C. (2008): "Robot-based hand motor therapy after stroke." Brain, Vol. 131, No. 2, pp. 425-437. https://doi.org/10.1093/brain/awm311

[9] Hurn, J., Kneebone, I., and Cropley, M. (2006): "Goal setting as an outcome measure: a systematic review." clinical rehabilitation, Vol. 20, pp. 756-72. https://doi.org/10.1177/ 0269215506070793

[10] Traczyk, M. (2016). The Making of a 3D Printed Rehabilitation Orthosis. http://blog.zmorph3d.com/3d-printed-rehabilitation-orthosis. Retrieved Jan 18, 2019.

[11] Young, James F. (2000): Basic mechanics. ELEC 201: Introduction to Engineering Design, Electrical and Computer Engineering Dept. Rice Univ.

[12] Ang, K. K., Sui, K., Soon, K., and Chin, Zheng yang. (2013): “A Randomized Controlled Trial on EEG-based motor imagery Brain-Computer Interface." Science and Engineering Research Council of Agency for Science, Technology and Re-search (A*STAR), Singapore, Vol. 7, pp. 1-35.

[13] Ang, K. K., and Guan, C. (2013): "Brain-computer interface in stroke rehabilitation.” Journal of Computing Science and Engineering, Vol. 7, No. 2, pp. 139-146.

[14] Bashivan, P., Rish, I., Yeasin, M., and Codella, N. (2016): "Learning Representations from EEG with Deep Recurrent-Convolutional Neural Networks.” International Conference on Learning Representations, pp. 1-15.

[15] Thodoroff, P., Pineau, J., \& Lim, A. (2016). Learning robust features using deep learning for automatic seizure detection. In Machine learning for healthcare conference (pp. 178190).

[16] Harvey, R. (2009): "Improving poststroke recovery: neuroplasticity and task-oriented training." Curr Treat Opt Cardio Med, Vol. 11, pp. 251-259. https://doi.org/10.1007/s11936009-0026-4

\section{$7 \quad$ Authors}

Emmanuel Osayande is with the Department of Electronic and Electrical Engineering, Obafemi Awolowo University, Ile-Ife Osun 220005, Nigeria.

Kayode P. Ayodele is with the Department of Electronic and Electrical Engineering, Obafemi Awolowo University, Ile-Ife Osun 220005, Nigeria.

Morenikeji A. Komolafe is with the Department of Medicine, Obafemi Awolowo University, Ile-Ife, Osun 220005, Nigeria.

Article submitted 2020-01-25. Resubmitted 2020-02-19. Final acceptance 2020-02-20. Final version published as submitted by the authors. 